\title{
On symbolic temporal information: Beliefs about the experience of duration
}

\author{
WITHOLD H. GALINAT \\ Universität Mannheim, Mannheim, West Germany \\ and \\ INGWER BORG \\ Justus-Liebig-Universität, Giessen, West Germany
}

\begin{abstract}
Judgments on the subjective duration of simple and complex imagined situations are studied. Four facets, concerning the evaluation of the situation (pleasant/unpleasant) and the characteristics of its events (many/few, variable/monotonous, difficult/easy), are taken into account. These facets proved significant for duration judgments in previous studies in which subjects were exposed to situations varying with respect to one of them. In this paper, we study whether these timeperception facets have comparable effects on the symbolic temporal basis of duration judgments, that is, whether they allow one to account for differences in the beliefs about duration experience in imagined situations. Two approaches were chosen: (1) Three groups of subjects compared the durations of all possible pairs of situations characterized by facet elments $x$ and $y$. The confusion probabilities could be scaled in one dimension for all groups. The facet elements defined intervals that were hierarchically nested. (2) The facets allowed us to distinguish 24 structuples. For each of them, a concrete situation was described in writing. Seventy-six subjects rated the subjective durations of the situations. The structuples led to a partial order that corresponded well to the duration ratings. Moreover, three of the facets showed significant main effects, and the fourth interacted significantly with two others. Finally, the facets were useful in explaining the similarity structure of the duration ratings.
\end{abstract}

There is a fairly large but somewhat confusing literature on the effects of so-called nontemporal variables on duration judgments. The varied findings are partly due to differences in experimental tasks (e.g., Falk \& Bindra, 1954; Ornstein, 1969; Schiff \& Thayer, 1968; Schiffman \& Bobko, 1974), different durations of the intervals to be estimated (e.g., Aschoff, 1985; Michon, 1975), different test paradigms (e.g., Hicks, Miller, \& Kinsbourne, 1976; Zakay \& Fallach, 1984), and different timejudgment methods (e.g., McConchie \& Rutschmann, 1971). Moreover, the vast majority of published research examines the effects of only one isolated nontemporal variable on duration judgments. Also, most researchers seem to assume that the observers' estimations result only from the perceptual input during the interval to be judged and from cognitive processes directly associated with it. Hence, the subjects' abstract knowledge about the subjective experience of duration and its deviation from objective time is neither considered nor controlled.

Recently, some researchers found it necessary to also take into account this abstract knowledge. For example, Fraisse (1981) distinguished between (1) perceived and/or memorized temporal information and (2) the knowledge of time (e.g., the knowledge of how much time is required

Address reprint requests to Ingwer Borg, Fachbereich Psychologie, Justus-Liebig-Universität, Otto-Behaghel-Strasse 10, 6300 Giessen, West Germany. to change a flat tire) and parachronometric references provided by the action performed during the interval (e.g., the number of pages read) as a basis for judgments of duration. He considers the former as memorized and the latter as symbolic temporal information. Similar distinctions have been proposed by Doob (1971) and Michon (1979) in their "primary versus secondary judgments" and "impressionist versus analytic strategy," respectively.

Arons and London (1969) demonstrated the usefulness of this distinction in a study on the effect of different levels of ego-involvement on duration judgments. The subjects were required to sort marbles by color. Under the high ego-involved condition, subjects were exposed to a wall mirror that simulated a one-way observation glass; under the low ego-involved condition, the same wall mirror was covered. After 7.5 min of marble sorting, the subjects were asked to estimate how many minutes the sorting had taken. Shortly afterward, the subjects were asked to indicate whether, in making their judgments, they altered their estimates based on their perceptual impressions of the time spent on sorting and to correct their original judgments. The results showed that $40 \%$ of the subjects did not report their actual perceptual impressions. Only after the subjects' corrections were taken into account did the data replicate the previous finding that time passes more quickly under conditions of increased ego-involvement (Meade, 1960; Rosenzweig \& Koht, 1933). 
Symbolic temporal information seems to influence duration judgments most under the following conditions: (1) the subject is motivated to judge the duration of an interval as accurately as possible in terms of objective time; (2) the subject has applicable knowledge about the duration of the events within the interval; and (3) the subject receives only relatively little and/or vague temporal information about the interval so that the knowledge structure must yield the necessary information for a judgment. These circumstances generally are met if the duration judgments are retrospective, if estimations of long intervals are required, if the interval happened long ago, if the duration of future and hypothetical events is estimated, and, most importantly, if the interval is filled not with artificial stimuli (e.g., metronome beats, light flashes, tones, word lists), but with natural events.

An aspect of symbolic temporal information not mentioned by Fraisse (1981) is that it may vary in its level of abstractness. It may consist simply of knowledge about the duration of events, reference to dates as given by calendars, or an interplay of dates and subjective experience (e.g., "I got married after my father's first heart attack last year"). On a higher level, it may be represented as parachronometric references provided by the event itself (e.g., "I read quite a number of pages; thus, a lot of time must have passed") and, even more abstractly, as beliefs about experienced and/or remembered duration. Such beliefs can serve to correct time judgments. For example, if the subject believes that time drags while performing a dull, repetitious task, he/she implicitly assumes that it appears subjectively longer than it actually is.

In the present study, we considered only beliefs about experienced duration. Two important questions can be asked in this context: Where do these beliefs come from, and what characteristics make them different from memorized temporal information in Fraisse's (1981) sense?

As to the origin of beliefs about experienced duration, we postulate that they mirror actual duration experiences stored in long-term memory. This postulate is supported by the results of a study by Block, Saggau, and Nickol (1983-84), who conducted a large survey on college students' beliefs concerning meanings and experiences of time. Among other things, they observed that beliefs about experienced duration are generally consistent with the empirical evidence on actual duration experience.

Since time is not perceived as an entity in itself, but rather is constituted by the physical and psychological events that occur within it (e.g., Gibson, 1975; Guyau, 1890 ), experience of duration is necessarily tied to nontemporal variables. Several of these variables (facets) are related to judgments on the duration of intervals (Galinat, 1984): (1) number of stimuli perceived during a given interval (e.g., Buffardi, 1971; Frankenhäuser, 1959; Mo, 1971; Ornstein, 1969); (2) amount of memory space required to store the events (e.g., Mulligan \& Schiffman, 1979; Ornstein, 1969; Schiffman \& Bobko, 1974, 1977); (3) difficulty (i.e., processing demands of the stimuli) (e.g., Avant, Lyman, \& Antes, 1975; Burnside, 1971; Hicks et al., 1976; Michon, 1965; Thomas \& Weaver, 1975; Vroon, 1970); (4) variability (i.e., the number of perceived changes of the stimuli) (Block \& Reed, 1978; Fraisse, 1963; Poynter, 1983; Poynter \& Homa, 1983); (5) evaluation (i.e., the perceived pleasantness of stimuli (e.g., Edmonds, Cahoon, \& Bridges, 1981; Harton, 1939; Langer, Wapner, \& Werner, 1961; Rosenzweig \& Koht, 1933); (6) degree of anticipatory tension toward the occurrence of an event (e.g., Block, George, \& Reed, 1980; Lordahl \& Berkowitz, 1975); (7) attentional selectivity during encoding and retrieving of the stimuli (Underwood, 1975; Underwood \& Swain, 1973) or attentional focusing on the passage of time itself (e.g., Curton \& Lordahl, 1974; Fraisse, 1963; McKay, 1977).

Other researchers have presented somewhat different lists of facets. For example, Loehlin (1959) factor analyzed duration judgments to identify four facets: (1) interest versus boredom (corresponding roughly to our variability and difficulty facets), (2) attention to time (corresponding to amount of attention), (3) repetition of an activity (corresponding to variability, difficulty, and number), (4) activity versus passivity (corresponding to number and storage space). Two more facets, stress (corresponding to evaluation) and goal approachment (corresponding to anticipatory tension), were not verified empirically. Although a stress effect was found in some studies (e.g., Falk \& Bindra, 1954; Langer et al., 1961), Schiff and Thayer (1968) explain their failure to find a similar effect by speculating that "extreme intensities [of this facet] may be required to produce significant effects on time judgments"' (p. 805). With respect to goal approachment, Loehlin (1959) admits that his investigation was not well suited to detect this facet because "it was desirable to make most of the tasks indefinite in length, i.e., comparable to ... only 'no goal' condition' (p. 18).

As to how beliefs about experienced duration and memorized temporal information, in Fraisse's (1981) sense, differ from each other, we assume that the latter may be retrieved from both short- and long-term memory, whereas the former can be retrieved only from long-term memory. In addition, memorized temporal information represents concrete event-sequence information (together with time cues) provided by the interval, whereas beliefs about experienced duration consist of generalized information based on prior experience (e.g., carrying out a repetitious uninteresting task makes time drag).

Little is known at present about the combined effect of more than one nontemporal facet on duration judgments. Studies that test more than one nontemporal facet at a time could provide information on the relative importance of these facets and their interactions. Such information would be valuable for a comprehensive theory of duration judgments. We probably lack this kind of knowledge because it is difficult to operationalize more than one facet in the laboratory and exert strict experimental control upon the facets. Therefore, different approaches may be needed. One such approach is the study of imagined situations. 
The present paper addresses beliefs about experienced duration, both in simple abstract comparisons and in complex situations varying in several facets simultaneously. This may provide some clues about the variables that determine how corrections of duration judgments are made and how they are interrelated. To know more about these variables represents an important and obvious requirement for a comprehensive theory of time judgments.

\section{EXPERIMENT 1}

We hypothesize that beliefs about experienced duration mirror people's actual duration experiences. To our knowledge, only two studies have investigated such beliefs. Henrikson (1948) examined beliefs about experienced duration and actual duration impression. He recorded both duration estimates and objective durations of impromptu speeches of subjects, as well as the subjectively experienced stage fright of each subject after his/her speech and the subject's opinion about how time seems to pass when a person is afraid. Ninety-five percent of the subjects thought that there is a positive correlation between stage fright and subjective duration of speaking time; however, the data showed the opposite trend. Block et al. (1983-84) studied students' beliefs about meanings and experiences of time in a large survey using 5-point rating scales. They observed, among other things, that a time interval seems to pass more slowly if it contains an unpleasant (as opposed to a pleasant) task, a task in which a person has little to do (as opposed to one in which the person is busy), a single task (as opposed to several different tasks), a task that takes place in an unchanging (as opposed to a changing) environment, and a task that is easy (as opposed to challenging). This corresponds well to results on actual duration judgments, indicating that intervals with unpleasant stimuli (e.g., Harton, 1939; Langer et al., 1961), many stimuli (e.g., Buffardi, 1971; Ornstein, 1969), variable stimuli (e.g., Block \& Reed, 1978; Poynter, 1983; Poynter \& Homa, 1983), and difficult stimuli (e.g., Burnside, 1971; Hicks et al., 1976; Zakay, Nitzan, \& Glicksohn, 1983) are judged to be longer than intervals with neutral or pleasant, few, less variable (i.e., more monotonous), and easy stimuli.

The question remains whether such findings can be replicated with different samples. Furthermore, the above rating results do not yet show that such data are all scalable in one dimension and, if so, in what model. We examined these issues in Experiment 1.

\section{Method}

Subjects. Three groups of subjects participated in this experiment: 38 college students of the Technische Hochschule Aachen, 56 college students of the Justus-Liebig-Universität Giessen, and 49 amateur Ping-Pong players of the Turnverein Birkenau. These groups will be denoted as Aa, Gi, and PP, respectively. The PingPong players worked in blue- or white-collar jobs and, on the average, were several years older than the students. All groups were composed of volunteers who did not receive pay or class credit for their participation.
Materials and Design. The pair comparison system was built on eight types of stimuli: pleasant, unpleasant, variable, monotonous, many, few, difficult, and easy. Hence, 28 comparisons were possible. For example, we could ask a subject whether he/she thinks that a pleasant situation is likely to be subjectively longer than a monotonous one. Concretely, all situations were phrased in terms of an activity. That is, test booklets with 28 pages were made in the following format:

Consider two situations, each characterized by one particular activity. Which one is likely to appear subjectively longer to you?

To do something for one hour that you do not like doing.

To do an awful lot for one hour.

The subjects had to choose one alternative (forced choice). Each page of the test booklet contained only one pair comparison. The order of the 28 possible pairings was determined randomly for the first booklet. The first pair comparison in booklet i was presented last in booklet $i+1$. In a second series of booklets, the order of the activities in each pair was swapped.

Procedure. The Aa and PP subjects were tested individually, whereas the Gi subjects were tested as a group. At the outset, the subjects were told,

In the following you will receive a test booklet. On each page of the booklet, you will be asked to decide which of two activities would appear longer to you. Please be sure to always pick one activity as the longer one, even if you feel that differences are minimal.

\section{Results}

The pair comparison data were aggregated over the individuals in each group. For all three groups, the resulting probabilities proved scalable in the sense of Thurstone's (1927) law of comparative judgment. We computed (as fit values) the correlations between the empirical probabilities and the corresponding probabilities reconstructed from the Thurstone scale values. We found $r$ (empirical, reconstructed) $=.9891$ for Group Gi, $r=.9893$ for Group Aa, and $r=.9906$ for Group PP. Moreover, the Gi, Aa, and PP scales turned out to be very similar, correlating no less than $r=.9768$ with each other.

Even though the assumptions of Thurstone's (1927) model seem justifiable in the present case, the scales do not depend on them. As long as the function that maps probabilities into distances is monotone, very similar scales result (e.g., Baird \& Noma, 1978). Thus, if the $p_{i j}$ probabilities are turned into dissimilarity scores $s_{i j}$, via $s_{i j}=\operatorname{abs}\left(p_{i j}-0.5\right)$, and if these $s_{i j}$ scores are scaled unidimensionally by ordinal multidimensional scaling, one obtains scales (Figure 1) that correlate highly with the Thurstone scales (minimal correlation is .9623). The scales have stress values of $\mathrm{S}(\mathrm{Gi})=9 \%, \mathrm{~S}(\mathrm{Aa})=13.2 \%$, and $\mathrm{S}(\mathrm{PP})=10 \%$, which lie substantially below the usual simulation norms for random data (Borg, 1981).

The orientation of the MDS scales with ends long and short follows directly from the data, since all three groups nested the situation characteristics relative to duration in the same hierarchical way. In all cases, pleasant and unpleasant are located at the ends of the scales. (For ease of comparison, the scales are all normed to the same length.) Within this interval, the pair many/few has the second largest distance. Then, within the interval defined 


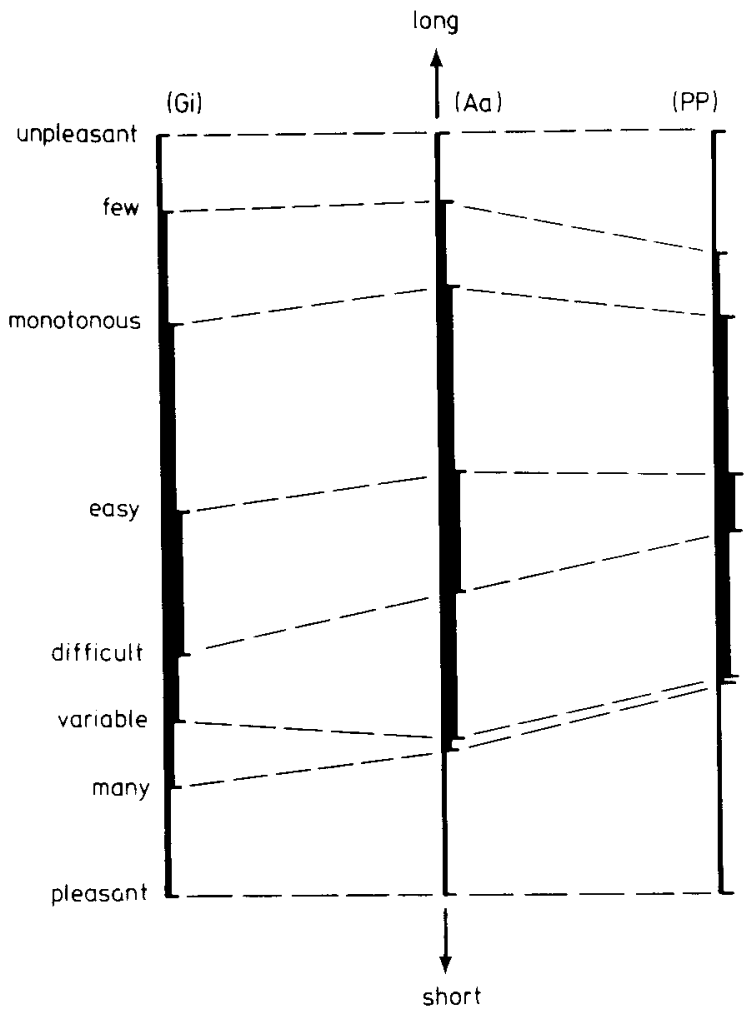

Figure 1. Scales for pair comparison data of subject Groups Gi, Aa, and PP; scales derived via ordinal MDS. Long and short refer to duration.

by many/few, we have the interval from monotonous to variable, and finally, within the monotonous/variable interval, the interval spanned by easy and difficult. This enables us to draw the scales in the nested way shown in Figure 1 .

Substantively, we see that the characteristics pleasant, many, variable, and difficult are on the short end of the scales, and unpleasant, few, monotonous, and easy are on the long end. Moreover, the data show that the facets can be ordered in their importance for duration judgments as pleasantness $>$ variability $>$ difficulty. Facet F4 (number) lies empirically between pleasantness and variability, but differs little from variability, particularly on the short end of the scale. Finally, we see that the characteristics difficult and easy had a very small differential effect on the duration evaluations. We return to this point later on.

\section{EXPERIMENT 2}

The duration judgment literature is replete with experiments that test the isolated effects of single facets, but there is-to our knowledge-no study that examines the conjoint effect of more than one facet at a time. This is true for actual duration judgments as well as for beliefs about experienced duration. In Experiment 2, we intended to gather knowledge about beliefs with respect to the conjoint influence of the facets studied in Experiment 1. We hypothesized that (1) the belief facets evaluation, number, variability, and difficulty all have an effect on duration judgments of imagined situations when they appear in conjunction; (2) situations with pleasant, many, variable, and difficult events are judged as shorter than imagined situations with unpleasant, few, monotonous, and easy events; (3) belief facets and their elements show the same ordering in terms of magnitude of effects on duration judgments as in Experiment 1.

\section{Method}

Subjects. Seventy-six subjects participated in Experiment 2: all 56 individuals from Group $\mathrm{Gi}$ and 20 individuals from Groups Aa and PP.

Materials and Design. The subjects received test booklets with descriptions of situations, such as the following:

Situation 8: You are working on the assembly line of a large manufacturer of electrical switches. Your job requires you to do the same thing over and over again, namely, to do many solderings on the switches that pass by on the assembly line. This task is very difficult, because the solderings have to be precisely in the locations marked on the switches. The speed of the assembly line is such that you have three minutes for one switch.

-How long did you feel that this task lasted? Did it appear longer or shorter than three minutes?

Situation 21: You are working as a secretary for the city. Your boss enters your office and puts a box onto your table. It contains some 200 letters. He asks you to quickly stamp a retum address on each of these letters, and then to carry everything over to the mailing office, so that all letters get into the mail today. You are not exactly excited about this very simple, but quite boring task. Nevertheless, you start right away to stamp a return address on each of the many letters. You need three minutes before you are done and ready to carry everything over to the mailing office, as you were asked to do.

-How long did you feel that this task lasted? Did it appear longer or shorter than three minutes?

These items were assessed on the following rating scale:

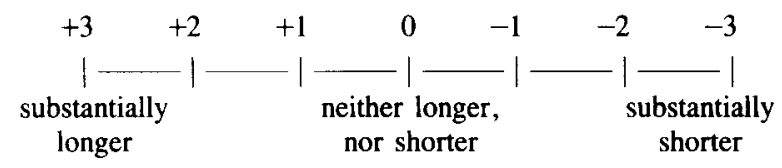

The 24 situations were derived from the structuples of the following four-faceted mapping sentence (Borg, 1979; Canter, 1984):

$$
\begin{aligned}
& \text { The respondent } \left.(r) \text { rates the } \begin{array}{c}
(1=\text { pleasant }) \\
(3=\text { neutral }) \\
(3=\text { unpleasant })
\end{array}\right) \text { situation with } \\
& (1=\text { many) }(1=\text { variable }) \\
& (2=\text { few }) \\
& \text { handle as }(2=\text { monotonous }) \\
& (+3=\text { difficult }) \\
& (-3=\text { very long in duration }) \\
& (2=\text { easy })
\end{aligned}
$$

The domain of the mapping sentence exhibits four facets, F1-F4. This implies that there are 24 different ways to read the mapping sentence. Hence, we can distinguish 24 different situations that had 
to be mapped on the range very long in duration to very short in duration by the subjects. Each situation is characterized by a particular combination of facet elements (structuple). For example, Situations 8 and 21 are constructed so that they possess the structuples neutral-monotonous-difficult-many and unpleasant-monotonouseasy-many, or simply 2211 and 3221 , respectively. To assign a particular structuple to a situation, several raters discussed the validity of the characterization of the situation's content. If agreement was reached, the situation was accepted for the study and its structuple was treated as a definition. The structuples of all 24 situations are shown in Table 1.

In addition to rating the duration of the 24 situations, the subjects also rated them with respect to experience ("How much personal experience do you have with this situation?') and empathy ("How well can you put yourself into the shoes of someone in this situation?'). Experience and empathy ratings were done on 7-point scales ranging from +3 (very much/very well) to -3 (very littlel very poorly).

Procedure. As in Experiment 1, Group Gi subjects were tested as a group, while Group Aa and Group PP subjects were tested individually. At the outset, all subjects were told,

You will be required to evaluate 24 complex situations with respect to how long they appear to last when experienced in reality, how much experience you have with them, and how well you can put yourself into the shoes of someone experiencing them. Each situation is described in writing on one page in these little booklets. Please read each situation carefully and try to project yourself into this situation before you make your evaluations! Please take care to make all three judgments before moving on to the next situation.

The order of the situations was balanced as before: For the first test booklet, the order was determined randomly; for booklet $i+1$, the last situation was the first of booklet $i$.

\section{Results}

After transforming the scores onto the interval $1, \ldots, 7$ ( -3 is mapped onto 1$)$, the statistics in Table 1 were ob-

Table 1

Statistics of Ratings for Situations with Respect to Subjective Duration, Experience, and Empathy

\begin{tabular}{|c|c|c|c|c|c|c|c|}
\hline \multirow[b]{2}{*}{ Situation } & \multirow[b]{2}{*}{ Structuple } & \multicolumn{2}{|c|}{ Duration } & \multicolumn{2}{|c|}{ Experience } & \multicolumn{2}{|c|}{ Empathy } \\
\hline & & Mean & $S D$ & Mean & $S D$ & Mean & $S D$ \\
\hline 1 & 1122 & 3.29 & 1.66 & 3.22 & 1.91 & 4.75 & 1.50 \\
\hline 2 & 2121 & 3.54 & 1.54 & 5.77 & 0.76 & 6.23 & 0.85 \\
\hline 3 & 1212 & 3.87 & 1.70 & 5.07 & 1.51 & 5.78 & 1.22 \\
\hline 4 & 1121 & 3.90 & 1.74 & 5.22 & 1.43 & 5.55 & 1.25 \\
\hline 5 & 1221 & 3.95 & 1.57 & 4.90 & 1.20 & 5.73 & 1.09 \\
\hline 6 & 1111 & 4.00 & 1.77 & 4.72 & 1.53 & 5.60 & 1.32 \\
\hline 7 & 2122 & 4.03 & 1.81 & 5.98 & 1.24 & 6.32 & 0.97 \\
\hline 8 & 2211 & 4.05 & 1.89 & 3.72 & 1.97 & 5.08 & 1.73 \\
\hline 9 & 1211 & 4.37 & 1.80 & 3.33 & 1.56 & 4.63 & 1.59 \\
\hline 10 & 1112 & 4.41 & 1.93 & 4.57 & 1.78 & 5.47 & 1.49 \\
\hline 11 & 2112 & 4.42 & 1.97 & 5.05 & 1.41 & 5.68 & 1.14 \\
\hline 12 & 1222 & 4.43 & 1.57 & 3.53 & 1.59 & 5.22 & 1.04 \\
\hline 13 & 3111 & 4.46 & 2.41 & 2.30 & 1.68 & 4.82 & 1.77 \\
\hline 14 & 2111 & 4.54 & 1.89 & 5.43 & 1.45 & 6.25 & 0.80 \\
\hline 15 & 2221 & 4.57 & 1.33 & 4.78 & 1.45 & 5.60 & 1.08 \\
\hline 16 & 3211 & 4.57 & 2.13 & 3.47 & 1.81 & 4.87 & 1.64 \\
\hline 17 & 2212 & 4.66 & 1.73 & 4.93 & 1.33 & 5.57 & 1.13 \\
\hline 18 & 3121 & 4.70 & 1.94 & 3.42 & 1.65 & 4.93 & 1.58 \\
\hline 19 & 3112 & 4.93 & 1.91 & 5.65 & 1.72 & 6.17 & 1.37 \\
\hline 20 & 3212 & 4.94 & 2.11 & 3.07 & 1.54 & 4.60 & 1.93 \\
\hline 21 & 3221 & 5.00 & 1.70 & 4.82 & 1.78 & 5.98 & 0.97 \\
\hline 22 & 2222 & 5.08 & 1.19 & 4.37 & 1.74 & 5.40 & 1.34 \\
\hline 23 & 3122 & 5.15 & 2.14 & 1.70 & 1.43 & 5.33 & 1.64 \\
\hline 24 & 3222 & 5.67 & 1.38 & 4.87 & 1.56 & 6.03 & 1.22 \\
\hline
\end{tabular}

Table 2

ANOVA Table for Duration Judgments

\begin{tabular}{lrrc}
\hline \multicolumn{1}{c}{ Source } & \multicolumn{1}{c}{ SS } & $d f$ & $p$ \\
\hline F1 & 237.10 & 2 & .001 \\
F2 & 39.67 & 1 & .001 \\
F3 & .03 & 1 & .928 \\
F4 & 36.78 & 1 & .001 \\
2-Way Interaction & 133.21 & 9 & .001 \\
F1 $\times$ F2 & 6.56 & 2 & .365 \\
F1 $\times$ F3 & 32.95 & 2 & .006 \\
F1 $\times$ F4 & 27.81 & 2 & .014 \\
F2 $\times$ F3 & 58.62 & 1 & .001 \\
F2 $\times$ F4 & 4.34 & 1 & .248 \\
F3 $\times$ F4 & 2.92 & 1 & .344 \\
3-Way Interaction & 28.96 & 7 & .261 \\
F1 $\times$ F2 $\times$ F3 & 14.17 & 2 & .114 \\
F1 $\times$ F2 $\times$ F4 & 1.86 & 2 & .752 \\
F1 $\times$ F3 $\times$ F4 & 1.72 & 2 & .768 \\
F2 $\times$ F3 $\times$ F4 & 11.21 & 1 & .064 \\
4-Way Interaction & 34.593 & 2 & .005 \\
Explained & 510.34 & 23 & .001 \\
Residual & 5858.54 & 1800 & \\
\hline
\end{tabular}

Note-F1 = evaluation facet; $F 2=$ variability facet; $F 3=$ difficulty facet; F4 $=$ number facet.

tained. We first note that the mean empathy values are all above 4 . Hence, the subjects indicated, on the average, that they had no problems putting themselves into the shoes of a person in any of the situations. This is true even if the subjects indicated (as in Situation 23) that they had no personal experience with this situation.

A simple test for the effects of the various facets was given by studying the mean duration ratings for a fixed facet element over all other facets. For example, we averaged the rating scores over all eight situations defined as pleasant, neutral, and unpleasant. We found that the pleasant situations led to an average duration value of 4.03, the neutral ones to 4.36 , and the unpleasant ones to 4.93. Thus, these averages are ordered just as the pair comparison data suggest: the more pleasant a situation (by definition), the shorter its subjective duration.

The mean duration scores for the other facets are also ordered as the scale values in Figure 1: mean duration values are 4.28 for variable, 4.59 for monotonous, 4.43 for difficult, 4.44 for easy, 4.30 for many, and 4.57 for few. Moreover, the difference in the averages for the elements of each facet mirror the hierarchical nesting in Figure 1. The largest effect is observed for Facet F1 (evaluation), the smallest for Facet F3 (difficulty).

A factorial analysis of variance with the Facets F1-F4 as factors shows highly significant main effects for Facets F1, F2 (variability), and F4 (number), but no significant effect for Facet F3 (see Table 2). Three of six two-way interactions also are significant, in addition to the fourway interaction. The four-way interaction is uninterpretable, but the other interactions provide interesting substantive insights. As shown above, Facet F3 has no significant main effect, but it apparently modulates Facets F1 and F2. Table 3 shows how: The duration of a positive/difficult situation seems longer than one that is posi- 
Table 3

Means for ANOVA Cells F1 $\times F 3, F 1 \times F 4$, and F2 $\times F 3$

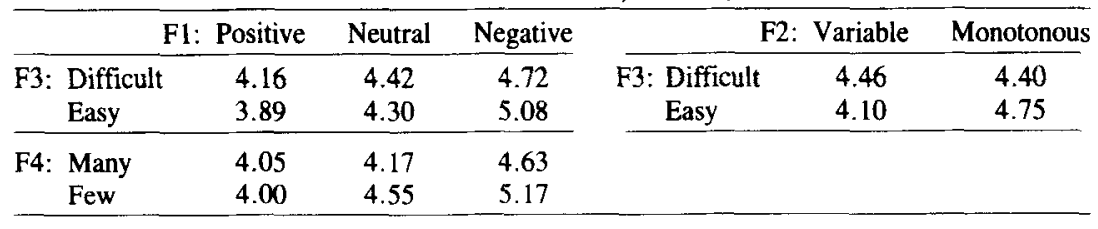

tive/easy, whereas the opposite is true if the situation is negative; similarly, a monotonous/easy situation seems longer than a monotonous/difficult one, whereas the opposite is true for a situation with variable stimuli. Also, the many/few distinction (Facet F4) interacts similarly with Facet F1 as difficult/easy does.

One might ask what and how much could be predicted if one formulates a theory that does not admit disordinal interactions of the facets. Since the facets can be ordered in the same substantive sense (i.e., from long to short), it is possible to bring the structuples into a partial order. That is, explicitly, we assume for Facet F1 that pleasant $<$ neutral < unpleasant, for Facet F2 that variable < monotonous, for Facet F3 that difficult < easy, and for Facet F4 that many $<$ few, where the symbol $<$ always means shorter than. If these assumptions are made without restrictions, disordinal interaction effects of the facets are excluded, and, without further assumptions, we can then decide for each pair of situations which of the two should be seen as longer, or that the two are incomparable. Let $S_{k}=\left(s_{1}, s_{2}, S_{3}, S_{4}\right)$ the situation $S_{k}$ as characterized by its structuple $s_{1} s_{2} s_{3} s_{4}$. Then, $S_{k} \leq S_{j}$ if and only if $s_{f k} \leq s_{f j}$, for all facets $f=1, \ldots, 4$. If, on the other hand, $s_{f k} \leq s_{f j}$ for some $f$, but not for others, then $S_{k}$ and $S_{j}$ are incomparable without making further assumptions on the weight of the various facets. Thus, for example, Situation 13 , which is characterized as 3111 , should be seen as shorter than Situation 23 , which has the structuple 3122 , because $S_{13}$ is not longer than $S_{23}$ in any facet, but is shorter with respect to the last two facets. In contrast, $S_{13}$ and $S_{15}$ are incomparable without further assumptions on the relative importance of the facets for duration judgments.

The partial order of the structuples that results under these conditions is shown in Figure 2. In the diagram, each situation $S$ can be compared to any other that can be reached from $S$ on a path that strictly leads up or down. Those situations that lie above $\mathrm{S}$ should be seen as shorter than $S$, and those below $S$ should be seen as longer than $S$. We use the mean duration values from Table 1 to test these predictions. The diagram shows confirmations as solid lines and violations as dashed lines. To unclutter the diagram, not all order tests are shown: if $S_{i} \leq S_{j}$ and $S_{j} \leq S_{k}$, then also $S_{i} \leq S_{k}$. Nevertheless, Figure 2 indicates that the predictions are confirmed well and, moreover, that violations of the predicted order relations occur primarily among the situations that were predicted to be subjectively short.

Analogous tests were carried out for each individual. There were altogether 162 order implications. On the average, 120 predictions were satisfied empirically. No standard statistical test is available to evaluate such a result against a null hypothesis of purely random effects. The usual way to provide some criteria anyway is to use computer simulations and check with what relative frequency the random data lead to rates of satisfaction of order predictions that reach or surpass those empirically observed (120 here). For such simulations, we took 1,000 vectors of 24 values from a rectangular distribution of the integers 1-9, and checked how these random profiles satisfied the order predictions. The simulations led to a negatively skewed distribution, with an average "correct" score of 100 . Ninety-three percent of the simulated values were less than the empirical average of 120 .

Two more classes of comparisons become possible if one assumes that the facets are ordered as F1 $<$ F2 $<$ F3 and F1 $<$ F4 F3. This implies that (1) If $s_{1 k}<s_{1 j}$ and $s_{f k}>s_{f j}$, for at most one $f=2, \ldots, 4$, then $S_{k} \leq S_{j}$; (2) if $s_{f k} \leq s_{f j}$, for $f=1, \ldots, 3$, with at least one strict

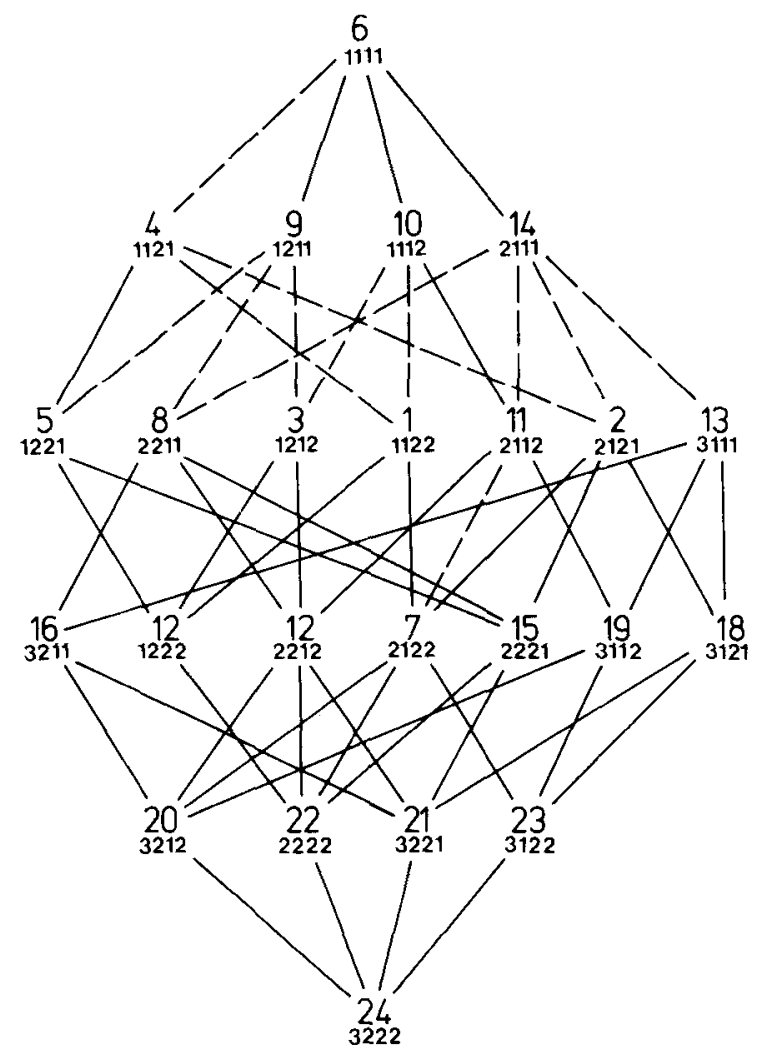

Figure 2. Hasse diagram of the 24 situations ordened by their structuples. Solid (broken) lines indicate where the respective average duration scores are (are not) ordered as predicted. 
inequality, and $s_{4 k}>s_{4 j}$, then $\mathrm{S}_{k} \leq \mathrm{S}_{j}$. This condition yields 276 testable predictions. Empirically, 193 of them are satisfied on the average. By simulation, we find that this value is greater than $94 \%$ of the random correct scores.

A slightly stronger test is given if we assume that Facets F2 and F4 have about equal weight so that the inequalities $s_{2 k}>s_{2 j}$ and $s_{4 k}<s_{4 j}$, and those with reversed signs, cancel each other. This condition yields 282 order predictions. On the average, 197 are satisfied, which is more than $94 \%$ of the simulated correct answers.

The above tests are quite weak, however, because they do not take into account how strongly the subjects felt that two situations, $S_{k}$ and $S_{j}$, differed in their duration. We repeated all tests and not only checked whether or not the empirical scores were ordered as predicted, but assessed the difference in the ascribed duration ratings. Under random conditions, the sum of such differences should be equal to zero. Empirically, we obtained for the three prediction conditions average difference scores of $78(11)$, 119 (17), and 122 (18), respectively, where the terms in parentheses give the standard errors of the statistics. Individually, about $80 \%$ of the subjects yielded positive sums of difference scores in all three cases. As before, all distributions are negatively skewed. Moreover, violations of the theory are observed mostly when short situations are involved.

We next turned to the empirical similarity of the situations with respect to subjective duration and asked whether Facets F1-F4 are useful to explain these similarities. We first represented, the correlations of the duration ratings of the 24 situations via ordinal MDS in a Euclidean space, and then checked whether the resulting configuration could be partitioned so that all points with a common element in Facet $F$ fell into one region and all other points into another.

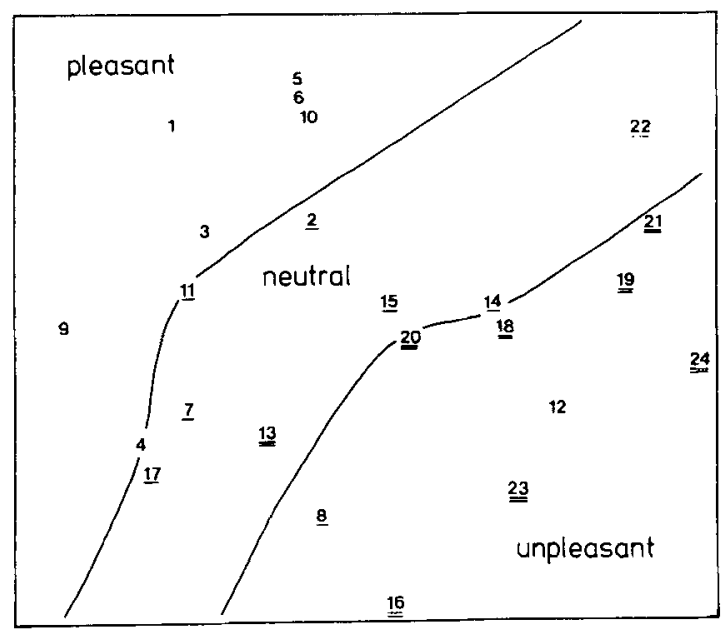

Figure 3. Plane spanned by principal components 1 and 2 in the four-dimensional MDS representation of the intercorrelations of duration ratings for the 24 situations. Border lines partition the plane relative to Facet F1. Numbers that are not/sing/y/doubly underlined represent pleasant/neutral/unpleasant situations, according to structuples in Table 1.

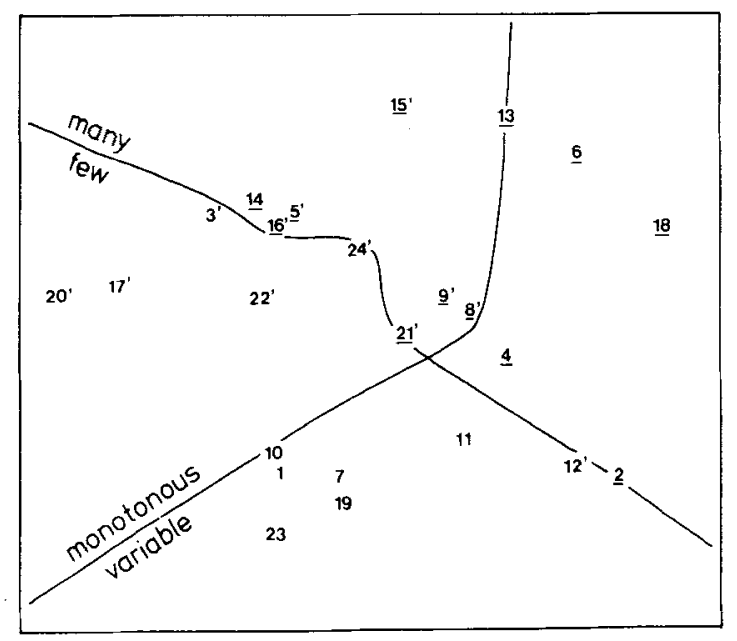

Figure 4. Plane spanned by principal components 3 and 4 in the four-dimensional MDS representation of the intercorrelations of the duration ratings for the 24 situations. Numbers underlined (not underlined) represent situations with many (few) events; numbers with (without) a prime represent situations with monotonous (variable) events, according to structuples in Table 1.

Since we were interested only in the order structure of the situations relative to their subjective durations, we computed monotone correlations (Guttman, 1981) over all 76 subjects for all pairs of data vectors. To represent these correlations by distances via MDS requires at least a four-dimensional space if one assumes that each combination of the four facets should lead to an independent discrimination function (Borg, 1986). It turns out that a four-dimensional MDS space also yields a good data fit (stress $=13 \%$ ). Figures 3 and 4 show the projection planes of this space spanned by the principal components 1-2 and 3-4, respectively.

In Figure 3, we discern a simple organization of points relative to their structuples: (1) those that represent pleasant situations fall into one region; (2) those that represent neutral situations into another; and (3) those that represent unpleasant situations into a third one. The three regions are disjoint and leave no points unclassified. The shown boundary lines remain, however, somewhat arbitrary with only 24 points in this plane. We have chosen them here such that they run roughly parallel. This entails that Points 13 and 8 lie in the wrong region, but these misplacements are quantitatively quite small. Only Point 12, which should fall into the pleasant region, is markedly misplaced. (In the four-dimensional space, this error is much reduced, however.)

Thus, we see that Facet F1 corresponds to a nontrivial organization of the empirical similarities. It is important that this regionalization is such that the neutral situations form a region between the pleasant and the unpleasant regions, because it is obviously a theoretical expectation that the elements of the evaluation facet are ordered.

In Figure 4, which shows an orthogonal space relative to Figure 3 , the points can be organized into regions in two ways, corresponding to the definitions made by Facets F2 and F4. The partitioning induced by Facet F4 (many- 
few) admits no errors at all, whereas the cut related to Facet F2 (monotonous-variable) leads to only two misclassifications. (One of them is Situation 12.) The border lines here and in Figure 3 form what is called a multiplex (Borg \& Lingoes, 1987), a primitive form of a Cartesian dimensional system.

It is thus plain that Facets F1, F2, and F4 allow us to discriminate almost perfectly among the 24 situations. Note that this is a much stronger finding than necessary to simply demonstrate effects of grouping variables (as in the usual discriminant analysis), because the respective distributions are virtually non-overlapping. We find, moreover, that Facet F3 (easy-difficult) does not lead to any discernible structuring in the MDS space. Since all situations vary with respect to all four facets, it thus seems that Facet F3 is too weak relative to the other three facets.

\section{GENERAL DISCUSSION}

Our basic theoretical assumption was that there is a double system of duration judgments, according to which duration judgments are, under some conditions, a direct function of the perceptual input processed during the corresponding interval, and, under other conditions, they are heavily influenced, if not almost exclusively determined, by symbolic temporal information stored in long-term memory.

In order to avoid misunderstandings, let us first point out what we have investigated and what we have not. We have not studied the interplay of the symbolic temporal system with its memorized counterpart. We also have not examined the circumstances under which we assume that symbolic temporal information is likely to become a viable component of duration judgments. What we have investigated is only one specific aspect of the symbolic temporal system: the content of people's beliefs about experienced duration. To this end, we have studied imagined situations, because the corresponding judgments represent a task in which no interplay between both parts of the double system can be assumed due to the lack of concrete, immediate memorized information. The content of people's beliefs was examined via four different facets: evaluation, number, variability, and difficulty. Experiment 1 revealed that each of the four facets, considered abstractly, was believed to influence duration experiences. Each facet had the expected short and long ends: Pleasant, many, variable, and difficult stimuli were assumed to make experienced duration shorter, whereas unpleasant, few, monotonous, and easy stimuli were thought to have the opposite effect.

The effects of the four facets were found to be quantitatively very different. In particular, people believed that it would not affect their duration experience very much if the stimuli in a situation are easy or difficult to handle, but that it would matter very much if the situation is evaluated as pleasant or unpleasant. The number and variability of the stimuli in a situation were believed to have an intermediate effect.
Essentially the same scales were found for three different groups of college students and nonstudents. These scales, derived from forced-choice pair comparison probabilities, correspond well to Block et al.'s (1983-84) rating scales. In particular, Block et al.'s facets were also ordered in terms of effect size as evaluation, number, variability, and difficulty.

Experiment 2 is also consistent with the prior findings on the effects of the four belief facets. This is even more remarkable, because the situations that had to be judged in Experiment 2 were much more complex and considerably less obtrusive with respect to the investigated facets than those used in previous studies.

Experiment 2 shows, in particular, that although the difficulty facet has no significant direct effect on duration judgments, it nevertheless moderates duration judgments: Difficulty dampens the effect of the pleasantness facet, whereas easiness accentuates it. There is a similar effect of difficult/easy on the variability facet. Hence, a situation that is considered difficult at least reduces the leverage of other belief facets on duration judgments. The analogous is true for the distinction many/few on the pleasantness facet: Many stimuli reduce the effect of positivity or negativity on duration judgments, whereas few stimuli accentuate it. These interactions might indicate, for example, that the subjects believe that a difficult situation leaves little room for effects of other facets, because the task absorbs all or most of the subjects' attention. A similar explanation may be true for many/few stimuli in a positive or negative context.

The partial-order hypothesis, which does not admit disordinal interactions, also proved fairly successful. Moreover, errors were not simply randomly scattered throughout the partial order, but occurred primarily among the short situations. This may simply mean that the short situations were not sufficiently different with respect to the facets studied here, or that further facets are needed for a better discrimination of short situations, or, of course, that disordinal interactions are particularly important for the short situations.

One may question the conclusions of Experiment 2, because all subjects first participated in Experiment 1, where the facets were used rather directly in the pair comparisons. Yet, the data from Experiment 1 proved very consistent, which makes it not very plausible that these facets were somehow learned during the experiment. Rather, the structure of the data suggests that a stable judgment system existed from the start. Moreover, this system is compatible with Block et al.'s (1983-84) findings. And finally, it does not appear very plausible that subjects were able to learn evaluation criteria in such a short time to such an extent that data structures such as those observed in Experiment 2-for situations with the complexity of those used in this experiment-are likely to result.

An interesting question concerns the relevance of the present findings for duration judgment research and theory. Prior research shows that subjects in duration judgment experiments can critically reflect their duration experiences and correct them for biases (Arons \& Lon- 
don, 1969). Our findings shed some light on what criteria might be used in such corrections. For example, if a worker feels that a lot of time has passed since he/she started a relatively uninteresting task, he/she may correct this impression by taking the belief into account that dull, easy, and unpleasant situations appear much longer than they actually are, and, thus, arrive at a duration judgment that is shorter than the one suggested by direct experience.

It is also noteworthy that the content of the beliefs studied is congruent with the results on actual duration judgments reported in the literature. For example, situations with many stimuli are thought to appear shorter than situations with only few stimuli. The same result is found with respect to actual duration judgments (e.g., Buffardi, 1971; Frankenhäuser, 1959; Ornstein, 1969). One reviewer remarked that there is an exception to this general congruence, because, according to Avant et al. (1975) and Thomas and Weaver (1975), low processing effort is associated with longer duration judgments than is high processing effort. Processing effort is roughly equivalent to our difficulty facet which, instead, shows that people think that difficult tasks appear subjectively shorter than easy ones. In other experiments, however, increases in processing effort have produced decreases in subjective duration estimates (e.g., Burnside, 1971; Hicks et al., 1976; Vroon, 1970; Zakay et al., 1983). It is interesting to note that the two groups of studies differ in that the former investigated durations of less than $100 \mathrm{msec}$, and the latter investigated intervals of more than $10 \mathrm{sec}$. Hence, one may speculate that intervals of less than $100 \mathrm{msec}$ may be too short to give rise to duration beliefs, either because no natural event occurs in such short intervals or because they are not comprehended as having a duration dimension.

Finally, if one assumes with us that beliefs about experienced duration mirror people's prior experience with the effects of nontemporal variables on duration judgments, then two further issues seem relevant for duration judgment theory and research: (1) Researchers should critically reflect why there was so much previous research on the effects of the difficulty facet, that is, the processing effort associated with encoding of and responding to stimuli presented in an interval. Judging from our data, this facet seems relatively unimportant. (2) At least some of the many nontemporal variables that affect duration judgments do interact. A comprehensive theory of duration judgment should take this into account.

\section{REFERENCES}

ARoNs, M., \& London, H. (1969). Correcting for compensation in studies of time estimation. Psychonomic Science, 17, 319-320.

AschoFf, J. (1985). On the perception of time during prolonged temporal isolation. Human Neurobiology, 4, 41-52.

Avant, L. L., Lyman, P. J., \& ANTEs, J. R. (1975). Effects of stimulus familiarity upon judged visual duration. Perception \& Psychophysics, 17, 253-262.

BAIRD, J. C., Noma, E. (1978). Fundamentals of scaling and psychophysics. New York: Wiley.
Block, R. A., George, E. J., \& ReED, M. A. (1980). A watched pot sometimes boils: A study of duration experience. Acta Psychologica, 46, 81-94.

BLOCK, R. A., REED, M. A. (1978). Remembered duration: Evidence for a contextual-change hypothesis. Journal of Experimental Psychology: Human Learning \& Memory, 4, 656-665.

Block, R. A., SAGgau, J. L., Nickol, L. H. (1983-84). Temporal inventory on meaning and experience: A structure of time. Imagination, Cognition \& Personality, 3, 203-225.

BoRG, I. (1979). Some basic concepts of facet theory. In J. C. Lingoes, E. E. Roskam, \& I. Borg (Eds.), Geometric representations of relational data (pp. 65-102). Ann Arbor, MI: Mathesis Press.

BORG, I. (1981). Anwendungsorientierte Multidimensionale Skalierung. Heidelberg: Springer.

Borg, I. (1986). Facettentheorie: Prinzipien und Beispiele. Psychologische Rundschau, 37, 121-137.

BORG, I., \& LINGOES, J. C. (1987). Multidimensional similarity structure analysis. New York: Springer.

BuFfardi, L. (1971). Factors affecting the filled-duration illusion in the auditory, tactual, and visual modalities. Perception \& Psychophysics, 10, 292-294.

BURNSIDE, W. (1971). Judgment of short time intervals while performing mathematical tasks. Perception \& Psychophysics, 10, 292-294.

CANTER, D. (Ed.). (1984). The facet approach to social research. New York: Springer.

Curton, E. D., \& Lohrdahl, D. S. (1974). Attentional focus and arousal in time estimation. Journal of Experimental Psychology, 103, 861-867.

DоOB, L. W. (1971). The patterning of time. New Haven, CT: Yale University Press.

Edmonds, E. M., CAHOON, D., \& BRIDGEs, B. (1981). The estimation of time as a function of positive, neutral, or negative expectancies. Bulletin of the Psychonomic Society, 17, 259-260.

FALK, J. L., \& BINDRA, D. (1954). Judgments of time as a function of serial position and stress. Journal of Experimental Psychology, 47, 279-282.

Fraisse, P. (1963). The psychology of time. London: Lowe \& Brydone.

Fraisse, P. (1981). Cognition of time in human activity. In G. d'Ydewalle \& W. Lens (Eds.), Cognition in motivation and learning (pp. 233-258). Hillsdale, NJ: Erlbaum.

FRANKENHÄUSER, M. (1959). Estimation of time: An experimental study. Stockholm: Almquist \& Wiksell.

Gauinat, W. H. (1984). Über ein sozio-kulturelles Modell zur Zeitschätzung. (Tech. Rep.). Aachen, West Germany: Psychologisches Institut

Gibson, J. J. (1975). Events are perceivable but time is not. In J. T. Fraser \& N. Lawrence (Eds.), The study of time II (pp. 295-301). New York: Springer.

GutTMan, L. (1981). Efficacy coefficients for differences among averages. In I. Borg (Ed.), Multidimensional data representations: When and why (pp. 1-10). Ann Arbor, MI: Mathesis Press.

GuYau, M. (1890). La genese de l'idee de temps. Paris: Alcan.

HarToN, J. J. (1939). An investigation of the influence of success and failure on the estimation of time. The Journal of General Psychology, 21, 51-62.

HENRIKSON, E. H. (1948). A study of stage fright and the judgment of speaking time. Joumal of Applied Psychology, 32, 532-536.

Hicks, R. E., Miller, G. W., Kinsbourne, M. (1976). Prospective and retrospective judgments of time as a function of amount of information processed. American Journal of Psychology, 89, 719-730.

LANGER, J., WAPNER, S., WERNER, H. (1961). The effect of danger upon the experience of time. American Journal of Psychology, 74, 94-97.

Lozhlin, J. C. (1959). The influence of different activities on the apparent length of time. Psychological Monographs, 73(4, Whole No. 474).

LORDAHL, D. S., \& Berkowitz, S. (1975). The watched pot does boil: A case of the wrong control group. Bulletin of the Psychonomic Society, 5, 45-46.

McConchie, R. D., \& Rutschmann, J. (1971). Human time estima- 
tion: On differences between methods. Perceptual \& Motor Skills, 32, 319-336.

McKay, T. (1977). Time estimation: Effects of attentional focus and a comparison of interval conditions. Perceptual \& Motor Skills, 45, 584-586.

Meade, R. (1960). Time estimates as affected by need tension and rate of progress. Journal of Psychology, 50, 173-177.

Michon, J. A. (1965). Studies on subjective duration: II. Subjective time measurement during tasks with different information content. Acta Psychologica, 24, 205-219.

Michon, J. A. (1975). Time experience and the memory process. In J. T. Fraser \& N. Lawrence (Eds.), The study of time II (p. 302-313). New York: Springer.

Michon, J. A. (1979). Le traitement de l'information temporelle. In P. Fraisse (Ed.), Du temps biologique au temps psychologique (pp. 264-296). Paris: P.U.F.

Mo, S. S. (1971). Judgment of temporal duration as a function of numerosity. Psychonomic Science, 24, 71-72.

Mulligan, R. M., \& Schiffman, H. R. (1979). Temporal experience as a function of organization in memory. Bulletin of the Psychonomic Society, 14, 417-420.

ORNSTEIN, R. E. (1969). On the experience of time. Hammondsworth, England: Penguin.

Poynter, W. D. (1983). Duration judgment and the segmentation of experience. Memory \& Cognition, 11, 77-82.

Poynter, W. D., \& Homa, D. (1983). Duration judgment and the experience of change. Perception \& Psychophysics, 33, 548-560.
Rosenzweig, S., \& KoHT, A. C. (1933). The experience of duration affected by need-tension. Joumal of Experimental Psychology, 16, 745-775.

SCHIFF, W., \& ThAYER, S. (1968). Cognitive and affective factors in temporal experience: Anticipated and experienced pleasant and unpleasant sensory events. Perceptual \& Motor Skills, 30, 895-902.

SCHIFfMaN, H. R., \& BoBKo, D. J. (1974). Effects of stimulus complexity on the perception of brief temporal intervals. Journal of $E x$ perimental Psychology, 103, 156-159.

Schiffman, H. R., \& BoвKo, D. J. (1977). The role of number and familiarity of stimuli in the perception of brief temporal intervals. American Journal of Psychology, 90, 85-93.

Thomas, E. A. C., \& Weaver, W. B. (1975). Cognitive processing and time perception. Perception \& Psychophysics, 17, 363-367.

Thurstone, L. L. (1927). A law of comparative judgment. Psychological Review, 34, 273-286.

UNDERWOOD, G. (1975). Attention and the perception of duration during encoding and retrieval. Perception, 4, 291-296.

UNDERWood, G., \& SwaIN, R. A. (1973). Selectivity of attention and the perception of duration. Perception, 2, 101-105.

VROON, P. A. (1970). Effects of presented and processed information on duration experience. Acta Psychologica, 34, 115-121.

ZAKAY, D., \& FALLACH, E. (1984). Immediate and remote time estimation: A comparison. Acta Psychologica, 57, 69-81.

Zakay, D., NitZan, D., \& Glicksohn, J. (1983). The influence of task difficulty and external tempo on subjective time estimation. Perception \& Psychophysics, 34, 451-456.

(Manuscript received June 17, 1985;

revision accepted for publication October 20, 1986). 